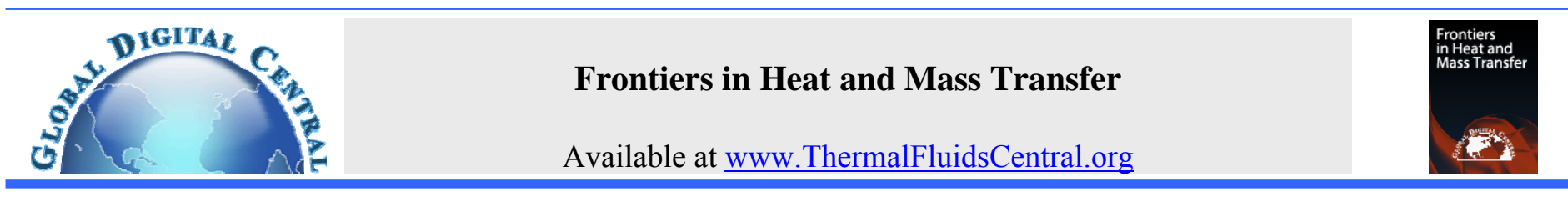

\title{
MHD CASSON VISCOUS DISSIPATIVE FLUID FLOW PAST A VERTICALLY INCLINED PLATE IN PRESENCE OF HEAT AND MASS TRANSFER: A FINITE ELEMENT TECHNIQUE
}

\author{
R. Srinivasa Raju ${ }^{\text {a, }}$, G. Jithender Reddy ${ }^{\mathrm{b}}$, G. Anitha ${ }^{\mathrm{a}}$ \\ ${ }^{a}$ Department of Mathematics, GITAM University, Hyderabad Campus, Rudraram, 502329, Telangana State, India \\ ${ }^{b}$ Department of Mathematics, VNR Vignana Jyothi Institute of Engineering and Technology, Hyderabad, Ranga Reddy (Dt), 500090, \\ Telangana State, India
}

\begin{abstract}
In the present study, consider an influence of chemical reaction on an unsteady MHD free convective, viscous dissipative Casson fluid flow over a vertically inclined plate in presence of magnetic field, heat and mass transfer. The modeling equations are converted to dimensionless equations, then solved through finite element technique. Computations were performed to analyze the behavior of fluid velocity, temperature, concentration and induced magnetic field on the inclined vertical plate with the variation of emerging physical parameters. Compared the present results with earlier reported studies for correctness and applicability of finite element technique. This model may be useful in view of lab experimental results for correctness and applicability and useful to analyze the fluid behavior in thermal engineering industries with the influence of the thermal, magnetic and chemical reaction effects etc.

Keywords: Magnetic Field, Casson Fluid Flow, Free Convection, Heat and Mass transfer, Finite Element Method.
\end{abstract}

\section{INTRODUCTION}

Non-Newtonian fluid theory is a part of fluid mechanics based on the continuum theory that a fluid particle may be considered as continuous in a structure. One of the non-newtonian fluids is a Pseudo plastic time independent fluid whose behaviour is that Viscosity decreases with increasing velocity gradient e.g. blood, polymer solutions, etc. Casson fluid is one of the pseudoplastic fluids that means shear thinning fluids (Casson, 1959). At low shear rates the shear thinning fluid is more viscous than the Newtonian fluid, and at high shear rates it is less viscous. So, MHD flow with Casson fluid is recently well-known. Harinath Reddy et al. (2016) studied the influence of radiation absorption and chemical reaction on unsteady magnetohydrodynamic free convective heat and mass transfer Casson fluid past an oscillating vertical plate embedded in a porous medium in the presence of constant wall temperature and concentration flow using finite difference method. Venkateswarlu and Satya Narayana (2016) studied the combined effect of Soret and Dufour on MHD flow of a Casson fluid past a stretching sheet in the presence of viscous dissipation, chemical reaction and variable thermal conductivity using shooting method. Rammohan Reddy et al. (2016) found the analytical solutions of MHD convective flow of a incompressible, viscoelastic, radiative, chemically reactive, electrically conducting and rotating fluid through a porous medium filled in a vertical channel in the presence of thermal diffusion using perturbation technique. Bhattacharyya (2013) found the numerical solutions of steady boundary layer stagnation-point flow of Casson fluid and heat transfer towards a shrinking/stretching sheet using very efficient shooting method. Mustafa and Khan (2015) investigated magnetic field effects on Casson nanofluid over nonlinearly stretching sheet. Nadeem et al. (2014) analyzed the steady stagnation point flow of a non-Newtonian fluid (Casson model) towards a stretching surface with heat transfer and Nano particles. Nadeem et al. (2013) explored three dimensional electrically conducting boundary layer flow of Casson fluid over stretching sheet saturated in a porous medium. Khalid et al. (2015) investigated the effects of magnetic field on free convection flow of Casson fluid over oscillating plate embedded in porous medium. Sharadha and Shankar (2015) studied boundary layer flow of Casson fluid over exponentially stretching sheet.

In the boundary layer, the influence of thermal radiation is relevant in many engineering problems because of its applications especially in high temperature engineering processes. The effect of heat radiation is important in controlling the quality of the final product as it affects the rate cooling. Due to the above fact, some of the authors have studied the effect of thermal radiation in their works, viz., Pal et al. (2013), Mukhopadhyay et al. (2011), Akbar et al. (2013), Bhattacharyya and his co-workers (2013) and (2012), Rashidi et al. (2014), Su et al. (2012). Ahmed and Mahdy (2016) deliberated the magnetohydrodynamic rotating flow of a laminar incompressible viscous electrically conducting fluid in the stagnation region of an impulsively rotating sphere in the presence of thermal radiation, heat and mass transfer effects. Prakash et al. (2014) studied the combined effects of induced magnetic field and radiation on MHD heat and mass transfer flow of viscous, incompressible, Newtonian fluid over a porous vertical plate in the presence of viscous and magnetic dissipation using perturbation technique. Raptis et al. (2003) studied the effect of radiation in an optically thin gray gas flowing past a vertical infinite plate in the presence of a magnetic field. Ahmed (2010) found the analytical solutions of induced magnetic field with radiating fluid over a porous vertical plate using Perturbation technique.

Therefore this work can be considered as extension of Prakash et al. (2014). So Novelty of this paper is discussion of numerical solutions

*Corresponding author. Email: srivass999@gmail.com 
using finite element technique of the unsteady natural convective Casson fluid flow past over an vertically inclined plate in the presence of a thermal radiation, chemical reaction, magnetic and viscous dissipations with induced magnetic field. Also, the study of grid independence of finite element technique is discussed through tabular form. The behaviors of different pertinent parameters on velocity, induced magnetic field, temperature and concentration profiles as well as Skin-friction, Rate of heat and mass transfer coefficients is discussed in detail.

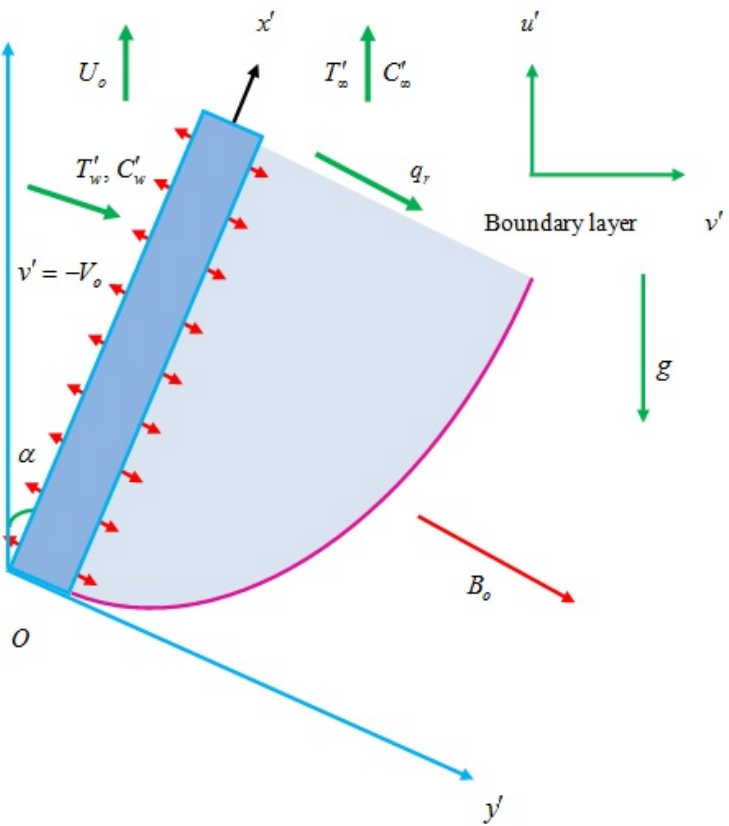

Fig. 1 Coordinate system and physical configuration

\section{MATHEMATICAL FORMULATION}

In this problem, we consider the effects of magnetic and viscous dissipation on steady two-dimensional magnetohydrodynamic mixed convective Casson fluid flow of a non-Newtonian and viscous incompressible radiative fluid over a vertical plate with induced magnetic field and heat and mass transfer. The coordinate system and the physical model of the problem are shown in Fig. 1.

For this present research work, we made the following assumptions:

i). In the fluid region, the plate is taken along $x^{\prime}$-axis in vertically upward direction and $y^{\prime}$-axis is normal to it.

ii). It is assumed that the wall is preserved at an unvarying temperature $T_{w}^{\prime}$ and concentration $C_{w}^{\prime}$ higher than the ambient temperature $T_{\infty}^{\prime}$ and concentration $C_{\infty}^{\prime}$ respectively.

iii). A uniform magnetic field of strength $B_{o}$ is assumed to be applied normal to the direction of flow.

iv). The magnetic Reynolds number of the flow is not taken to be small and hence the induced magnetic field is not insignificant.

v). The thermal diffusion and diffusion thermo effects are neglected due to the concentration of the diffusing species is assumed to be very small in comparison with the other chemical species.

vi). All the fluid properties are assumed to be constant except the effect of the pressure gradient in the body force term.

vii). There is a first order chemical reaction between the diffusing species and the fluid.

viii). Since the flow is assumed to be in the direction of $x^{\prime}$-axis, therefore all the physical quantities are functions of space coordinates in $y^{\prime}$ only. ix). Consider viscous dissipation in the energy equation.

The rheological equation of state for the Cauchy stress tensor of Casson fluid (Dash et al. (1996)) is written as

$\tau=\tau_{0}+\mu \alpha^{*}$

equivalently $\tau_{i j}=\left\{\begin{array}{l}2\left(\mu_{B}+\frac{p_{y}}{\sqrt{2 \pi}}\right) e_{i j}, \pi>\pi_{c} \\ 2\left(\mu_{B}+\frac{p_{y}}{\sqrt{2 \pi_{c}}}\right) e_{i j}, \pi<\pi_{c}\end{array}\right.$

where $\tau$ is shear stress, $\tau_{0}$ is Casson yield stress, $\mu$ is dynamic viscosity, $\alpha^{*}$ is shear rate, $\pi=e_{i j} e_{i j}$ and $e_{i j}$ is the $(i, j)^{\text {th }}$ component of deformation rate, $\pi$ is the product based on the non-Newtonian fluid, $\pi_{c}$ is a critical value of this product, $\mu_{B}$ is plastic dynamic viscosity of the non-Newtonian fluid,

$$
p_{y}=\frac{\mu_{B} \sqrt{2 \pi}}{\gamma}
$$

denote the yield stress of fluid.

Some fluids require a gradually increasing shear stress to maintain a constant strain rate and are called Rheopectic, in the case of Casson fluid (Non-Newtonian) flow where $\pi>\pi_{c}$

$$
\mu=\mu_{B}+\frac{p_{y}}{\sqrt{2 \pi}}
$$

Substituting Eq. (3) into Eq. (4), then, the kinematic viscosity can be written as

$v=\frac{\mu}{\rho}=\frac{\mu_{B}}{\rho}\left(1+\frac{1}{\gamma}\right)$

Finally $\gamma$ is the Casson fluid parameter and as $\gamma \rightarrow \infty$, the governing equations of the Casson fluid model $(\gamma \nrightarrow \infty)$ given by Eqs. (8)-(11) become the governing equations of the Newtonian fluid model $(\gamma \rightarrow \infty)$.

Then under usual Boussinesq's approximation along with the assumptions considered to the flow, the fundamental governing partial differential equations that illustrate the physical situation are given by Equation of Conservation of Electric Charge:

$\nabla \bullet \bar{J}=0$ where $\bar{J}=\left(J_{x}, J_{y}, J_{z}\right)$

Gauss Law of Magnetism:

$\frac{\partial H_{y}^{\prime}}{\partial y^{\prime}}=0 \Rightarrow H_{y}^{\prime}=B_{o}$ (a constant)

Equation of Conservation of Momentum:

$v^{\prime} \frac{\partial u^{\prime}}{\partial y^{\prime}}=v\left(\frac{1+\gamma}{\gamma}\right) \frac{\partial^{2} u^{\prime}}{\partial y^{\prime 2}}+g \beta\left(T^{\prime}-T_{\infty}^{\prime}\right)(\cos \psi)+$

$g \beta^{*}\left(C^{\prime}-C_{\infty}^{\prime}\right)(\cos \psi)+\left(\frac{\mu_{e} B_{o}}{\rho}\right) \frac{\partial H_{x}^{\prime}}{\partial y^{\prime}}$

Equation of Conservation of Energy:

$v^{\prime} \frac{\partial T^{\prime}}{\partial y^{\prime}}=\left(\frac{\kappa}{\rho C_{p}}\right) \frac{\partial^{2} T^{\prime}}{\partial y^{\prime 2}}-\left(\frac{1}{\rho C_{p}}\right) \frac{\partial q_{r}}{\partial y^{\prime}}+\frac{v}{C_{p}}\left(\frac{\partial u^{\prime}}{\partial y^{\prime}}\right)^{2}$

$+\left(\frac{1}{\sigma \rho C_{p}}\right)\left(\frac{\partial H_{x}^{\prime}}{\partial y^{\prime}}\right)^{2}$

Equation of Conservation of Magnetic Induction:

$v^{\prime} \frac{\partial H_{x}^{\prime}}{\partial y^{\prime}}=\left(\frac{1}{\sigma \mu_{e}}\right) \frac{\partial^{2} H_{x}^{\prime}}{\partial y^{\prime 2}}+B_{o}\left(\frac{\partial u^{\prime}}{\partial y^{\prime}}\right)$ 
Species Diffusion Equation:

$v^{\prime} \frac{\partial C^{\prime}}{\partial y^{\prime}}=D \frac{\partial^{2} C^{\prime}}{\partial y^{\prime 2}}-k_{r}^{\prime}\left(C^{\prime}-C_{\infty}^{\prime}\right)$

subject to the appropriate boundary conditions (Prakash et al. 2014)

$u^{\prime}=0, \quad v^{\prime}=-V_{o}, \quad T^{\prime}=T_{w}^{\prime}, \quad C^{\prime}=C_{w}^{\prime}, \quad H_{x}^{\prime}=0 \quad$ at $y^{\prime}=0$

$u^{\prime} \rightarrow U_{o}, \quad T^{\prime} \rightarrow T_{\infty}^{\prime}, \quad C^{\prime} \rightarrow C_{\infty}^{\prime}, \quad H_{x}^{\prime} \rightarrow 0$ as $\left.y^{\prime} \rightarrow \infty \quad\right\}$

In case of an optically thin gray gas, the expression for local radiant (Prakash et al. 2014) is given by

$\frac{\partial q_{r}}{\partial y^{\prime}}=-4 a \sigma^{*}\left(T_{\infty}^{\prime 4}-T^{\prime 4}\right)$

It is assumed that the temperature differences within the flow are sufficiently small and that $T^{\prime 4}$ may be expressed as a linear function of the temperature. This is obtained by expanding $T^{\prime 4}$ in a Taylor series about $T_{\infty}^{\prime}$ and neglecting the higher order terms, thus we get (Prakash et al. 2014)

$T^{\prime 4} \cong 4 T_{\infty}^{\prime 3} T^{\prime}-3 T_{\infty}^{\prime 4}$

Using the following non-dimensional quantities (15)

$u=\frac{u^{\prime}}{U_{o}}, y=\frac{y^{\prime} V_{o}}{v}, B=\frac{H_{x}^{\prime}}{U_{o}}, \theta=\frac{T^{\prime}-T_{\infty}^{\prime}}{T_{w}^{\prime}-T_{\infty}^{\prime}}, \phi=\frac{C^{\prime}-C_{\infty}^{\prime}}{C_{w}^{\prime}-C_{\infty}^{\prime}}$,

$M=\frac{\mu_{e} B_{o}}{\rho V_{o}}, G r=\frac{v g \beta\left(T_{w}^{\prime}-T_{\infty}^{\prime}\right)}{U_{o} V_{o}^{2}}, G m=\frac{v g \beta\left(C_{w}^{\prime}-C_{\infty}^{\prime}\right)}{U_{o} V_{o}^{2}}$,

$\operatorname{Pr}=\frac{v \rho C_{p}}{\alpha}, S c=\frac{v}{D}, \operatorname{Re}=\frac{U_{o} x^{\prime}}{v}, \operatorname{Pr} m=\sigma v \mu_{e}$

and with the help of Eqs. (13) and (14), the governing Eqs. (8)-(11) reduce to

$\left(1+\frac{1}{\gamma}\right) \frac{d^{2} u}{d y^{2}}+\frac{d u}{d y}+M \frac{d B}{d y}+G r(\cos \psi) \theta+G c(\cos \psi) \phi=0$

$\frac{d^{2} \theta}{d y^{2}}+(\operatorname{Pr}) \frac{d \theta}{d y}-\frac{(R)(\operatorname{Pr})}{4} \theta+(E c)(\operatorname{Pr})\left(\frac{d u}{d y}\right)^{2}$

$+\frac{(E c)(\operatorname{Pr})}{\operatorname{Pr} m}\left(\frac{d B}{d y}\right)^{2}=0$

$\frac{d^{2} B}{d y^{2}}+(M)(\operatorname{Pr} m) \frac{d u}{d y}+(\operatorname{Pr} m) \frac{d B}{d y}=0$

$\frac{d^{2} \phi}{d y^{2}}+(S c) \frac{d \phi}{d y}-(K r)(S c) \phi=0$

with associated initial and boundary conditions

$$
\left.\begin{array}{l}
u=0, \theta=1, \phi=1, B=0 \text { at } y=0 \\
u \rightarrow 1, \theta \rightarrow 0, \phi \rightarrow 0, B \rightarrow 0 \text { as } y \rightarrow \infty
\end{array}\right\}
$$

All the symbols are defined in nomenclature. The mathematical statement of the problem is now complete and embodies the solution of Eqs. (16), (17), (18) and (19) subject to boundary conditions (20). For practical engineering applications and the design of chemical engineering systems, the local skin-friction, Nusselt number and Sherwood number are important physical parameters for this type of boundary layer flow. The Skin-friction at the plate, which in the nondimensional form is given by

$C_{f}=\left(1+\frac{1}{\gamma}\right) \frac{\tau_{w}^{\prime}}{\rho U_{o} v}=\left(1+\frac{1}{\gamma}\right)\left(\frac{\partial u}{\partial y}\right)_{y=0}$

The rate of heat transfer coefficient, which in the non-dimensional form in terms of the Nusselt number is given by
$N u=-x^{\prime} \frac{\left(\frac{\partial T^{\prime}}{\partial y^{\prime}}\right)_{y^{\prime}=0}}{T_{w}^{\prime}-T_{\infty}^{\prime}} \Rightarrow N u \operatorname{Re}^{-1}=-\left(\frac{\partial \theta}{\partial y}\right)_{y=0}$

The rate of mass transfer coefficient, which in the nondimensional form in terms of the Sherwood number, is given by

$S h=-x^{\prime} \frac{\left(\frac{\partial C^{\prime}}{\partial y^{\prime}}\right)_{y^{\prime}=0}}{C_{w}^{\prime}-C_{\infty}^{\prime}} \Rightarrow S h \operatorname{Re}^{-1}=-\left(\frac{\partial \phi}{\partial y}\right)_{y=0}$

\section{NUMERICAL SOLUTIONS BY FEM}

3. 1. Finite Element Method (FEM): The finite element method (FEM) is a numerical and computer based technique of solving a variety of practical engineering problems that arise in different fields such as, in heat transfer, fluid mechanics (Srinivasa Raju et al. 2015; Bhargava and Rana, 2011; Sheri and Raju, 2015, Sheri and Raju, 2016, Sivaiah and Raju, 2013; Srinivasa Raju et al. 2016), chemical processing (Lin and Lo, 2003)), rigid body dynamics (Dettmer and Peric, 2006), solid mechanics (Hansbo and Hansbo, 2003), and many other fields. It is recognized by developers and users as one of the most powerful numerical analysis tools ever devised to analyze complex problems of engineering. The sophistication of the method, its accuracy, simplicity, and computability all make it a widely used tool in the engineering modeling and design process. It has been applied to a number of physical problems, where the governing differential equations are solved by transforming them into a matrix equation. The primary feature of FEM is its ability to describe the geometry or the media of the problem being analyzed with great flexibility. This is because the discretization of the domain of the problem is performed using highly flexible uniform or non uniform patches or elements that can easily describe complex shapes. The method essentially consists, assuming the piecewise continuous function for the solution and obtaining the parameters of the functions in a manner that reduces the error in the solution. An excellent description of finite element formulations is available in Bathe (1996) and Reddy (1985). The steps involved in the finite element analysis areas follows.

3. 1. 1. Discretization of the Domain: The basic concept of the FEM is to divide the domain or region of the problem into small connected patches, called finite elements. The collection of elements is called the finite element mesh. These finite elements are connected in a non overlapping manner, such that they completely cover the entire space of the problem.

\section{1. 2. Generation of the Element Equations:}

- A typical element is isolated from the mesh and the variational formulation of the given problem is constructed over the typical element.

- Over an element, an approximate solution of the variational problem is supposed, and by substituting this in the system, the element equations are generated.

- The element matrix, which is also known as stiffness matrix, is constructed by using the element interpolation functions.

These steps result in a matrix equation of the form $\left\{K^{e}\right\}\left\{u^{e}\right\}=\left\{F^{e}\right\}$ which defines the finite element model of the original equation.

3. 1. 3. Assembly of the Element Equations: The algebraic equations so obtained are assembled by imposing the inter element continuity conditions. This yields a large number of algebraic equations known as the global finite element model, which governs the whole domain. 
3. 1. 4. Imposition of the Boundary Conditions: On the assembled equations, the Dirichlet's and Neumann boundary conditions (20) are imposed.

3. 1. 5. Solution of Assembled Equations: The assembled equations so obtained can be solved by any of the numerical techniques, namely, Gauss elimination method, LU decomposition method, and the final matrix equation can be solved by a direct or indirect (iterative) method. For computational purposes, the coordinate $y$ is varied from 0 to $y_{\max }=9$, where $y_{\max }$ represents infinity $i$.e., external to the momentum, energy and concentration boundary layers. The whole domain is divided into a set of 90 line segments of equal width 0.1 , each element being two-noded.

\subsection{Variational formulation:}

The variational formulation associated with Eqs. (16)-(19) over a typical two-noded linear element $\left(y_{e}, y_{e+1}\right)$ is given by

$\int_{y_{e}}^{y_{e+1}} w_{1}\left[\begin{array}{l}\left(1+\frac{1}{\gamma}\right) \frac{d^{2} u}{d y^{2}}+\frac{d u}{d y}+M \frac{d B}{d y}+G r(\cos \psi) \theta \\ +G c(\cos \psi) \phi\end{array}\right] d y=0$

$\int_{y_{e}}^{y_{e+1}} w_{2}\left[\begin{array}{l}4(\operatorname{Pr} m) \frac{d^{2} \theta}{d y^{2}}+4(\operatorname{Pr} m)(\operatorname{Pr}) \frac{d \theta}{d y}-(\operatorname{Pr} m)(R)(\operatorname{Pr}) \theta \\ +4(\operatorname{Pr} m)(E c)(\operatorname{Pr})\left(\frac{d u}{d y}\right)^{2}+4(E c)(\operatorname{Pr})\left(\frac{d B}{d y}\right)^{2}\end{array}\right] d y=0$

$\int_{y_{e}}^{y_{e+1}} w_{3}\left[\frac{d^{2} B}{d y^{2}}+(M)(\operatorname{Pr} m) \frac{d u}{d y}+(\operatorname{Pr} m) \frac{d B}{d y}\right] d y=0$

$\int_{y_{e}}^{y_{e+1}} w_{4}\left[\frac{d^{2} \phi}{d y^{2}}+(S c) \frac{d \phi}{d y}-(K r)(S c) \phi\right] d y=0$

Where $w_{1}, w_{2}, w_{3}, w_{4}$ are arbitrary test functions and may be viewed as the variation in $u, \theta, B$ and $\phi$ respectively. After reducing the order of integration and non-linearity, we arrive at the following system of equations

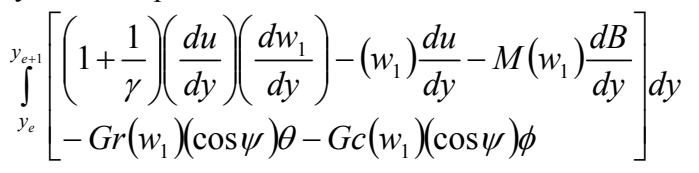

$-\left[\left(w_{1}\right)\left(1+\frac{1}{\gamma}\right)\left(\frac{\partial u}{\partial y}\right)\right]_{y_{e}}^{y_{e+1}}=0$

$\int_{y_{e}}^{y_{e+1}}\left[\begin{array}{c}4(\operatorname{Pr} m)(\operatorname{Pr})\left(w_{2}\right)\left(\frac{\partial \theta}{\partial y}\right)+4(\operatorname{Pr} m)\left(\frac{\partial w_{2}}{\partial y}\right)\left(\frac{\partial \theta}{\partial y}\right) \\ -4(\operatorname{Pr} m)(E c)(\operatorname{Pr})\left(w_{2}\right)\left(\frac{\partial \bar{u}}{\partial y}\right)\left(\frac{\partial u}{\partial y}\right) \\ -4(E c)(\operatorname{Pr})\left(w_{2}\right)\left(\frac{\partial \bar{B}}{\partial y}\right)\left(\frac{\partial B}{\partial y}\right) \\ +(\operatorname{Pr} m)(\operatorname{Pr})(R)\left(w_{2}\right) \theta\end{array}\right] d y$

$$
-\left[4(\operatorname{Pr} m)\left(w_{2}\right)\left(\frac{\partial \theta}{\partial y}\right)\right]_{y_{e}}^{y_{e+1}}=0
$$

$$
\begin{gathered}
\int_{y_{e}}^{y_{e+1}}\left[\begin{array}{l}
\left(\frac{d B}{d y}\right)\left(\frac{d w_{3}}{d y}\right)-M\left(w_{3}\right)(\operatorname{Pr} m) \frac{d u}{d y} \\
-(\operatorname{Pr} m)\left(w_{3}\right) \frac{d B}{d y}
\end{array}\right] d y-\left[\left(w_{3}\right)\left(\frac{\partial B}{\partial y}\right)\right]_{y_{e}}^{y_{e+1}}=0 \\
\int_{y_{e}}^{y_{e+1}}\left[\begin{array}{l}
\left(\frac{d \phi}{d y}\right)\left(\frac{d w_{4}}{d y}\right)-(S c)\left(w_{4}\right) \frac{d \phi}{d y} \\
+(K r)(S c)\left(w_{4}\right) \phi
\end{array}\right] d y-\left[\left(w_{4}\right)\left(\frac{\partial \phi}{\partial y}\right)\right]_{y_{e}}^{y_{e+1}}=0
\end{gathered}
$$

3.3 Finite Element formulation:

The finite element model may be obtained from Eqs. (28)-(31) by substituting finite element approximations of the form:

$$
u=\sum_{j=1}^{2} u_{j}^{e} \psi_{j}^{e}, \theta=\sum_{j=1}^{2} \theta_{j}^{e} \psi_{j}^{e}, \quad B=\sum_{j=1}^{2} B_{j}^{e} \psi_{j}^{e}, \phi=\sum_{j=1}^{2} \phi_{j}^{e} \psi_{j}^{e},
$$

With $w_{1}=w_{2}=w_{3}=w_{4}=\psi_{i}^{e}(i=1,2) \quad$ where $u_{j}^{e}, \theta_{j}^{e}, \quad B_{j}^{e}$ and $\phi_{j}^{e}$ are the Velocity, temperature, induced magnetic field and concentration respectively at the $j^{\text {th }}$ node of typical $e^{\text {th }}$ element $\left(y_{e}, y_{e+1}\right)$ and $\psi_{i}^{e}$ are the shape functions for this element $\left(y_{e}, y_{e+1}\right)$ and are taken as:

$\psi_{1}^{e}=\frac{y_{e+1}-y}{y_{e+1}-y_{e}}$ and $\psi_{2}^{e}=\frac{y-y_{e}}{y_{e+1}-y_{e}}, y_{e} \leq y \leq y_{e+1}$

The finite element model of the equations for $e^{\text {th }}$ element thus formed is given by

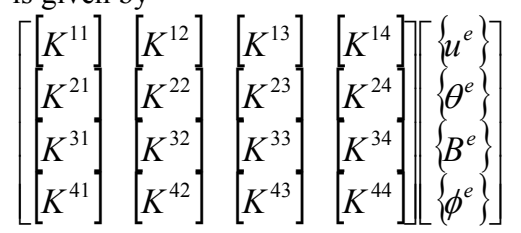

$+\left[\begin{array}{l}{\left[M^{11}\right.} \\ M^{21} \\ {\left[M^{31}\right.} \\ M^{41}\end{array}\right]\left[\begin{array}{l}M^{12} \\ M^{22}\end{array}\right]\left[\begin{array}{l}M^{13} \\ M^{32} \\ M^{23}\end{array}\right]\left[\begin{array}{l}M^{14} \\ M^{33} \\ M^{43}\end{array}\right]\left[\begin{array}{l}M^{24} \\ M^{34} \\ M^{44}\end{array}\right]\left[\begin{array}{l}\left\{u^{\prime e}\right\} \\ \left.\theta^{\prime e}\right\} \\ B^{\prime e} \\ \left.\phi^{\prime e}\right\}\end{array}\right]$

$$
=\left[\begin{array}{l}
\left\{b^{1 e}\right. \\
b^{2 e} \\
b^{3 e} \\
b^{4 e}
\end{array}\right\}
$$

Where $\left\{\left[K^{m n}\right],\left[M^{m n}\right]\right\},\left\{u^{e}\right\},\left\{\theta^{e}\right\},\left\{B^{e}\right\},\left\{\phi^{e}\right\},\left\{u^{\prime e}\right\}$,

$\left\{\theta^{\prime e}\right\},\left\{B^{\prime e}\right\},\left\{\phi^{\prime e}\right\}$ and $\left\{b^{m e}\right\},(m, n=1,2,3,4)$ are the set of matrices defined as follows:

$$
\begin{aligned}
& K_{i j}^{11}=\left(1+\frac{1}{\gamma}\right) \int_{y_{e}}^{y_{e+1}}\left(\frac{\partial \psi_{i}^{e}}{\partial y}\right)\left(\frac{\partial \psi_{j}^{e}}{\partial y}\right) d y-\int_{y_{e}}^{y_{e+1}}\left(\psi_{i}^{e}\right)\left(\frac{\partial \psi_{j}^{e}}{\partial y}\right) d y, \\
& K_{i j}^{12}=-M \int_{y_{e}}^{y_{e+1}}\left(\psi_{i}^{e}\right)\left(\frac{\partial \psi_{j}^{e}}{\partial y}\right) d y, K_{i j}^{13}=-(G r+G c) \int_{y_{e}}^{y_{e+1}}\left(\psi_{i}^{e}\right)\left(\psi_{j}^{e}\right) d y, \\
& K_{i j}^{14}=K_{i j}^{24}=K_{i j}^{33}=K_{i j}^{34}=K_{i j}^{43}=K_{i j}^{44}=0,
\end{aligned}
$$




$$
\begin{aligned}
& M_{i j}^{11}=M_{i j}^{22}=M_{i j}^{33}=M_{i j}^{44}=\int_{y_{e}}^{y_{e+1}}\left(\psi_{i}^{e}\right)\left(\psi_{j}^{e}\right) d y, \\
& M_{i j}^{12}=M_{i j}^{13}=M_{i j}^{14}=M_{i j}^{21}=M_{i j}^{23}=M_{i j}^{24}= \\
& M_{i j}^{31}=M_{i j}^{32}=M_{i j}^{34}=M_{i j}^{41}=M_{i j}^{42}=M_{i j}^{43}=0, \\
& K_{i j}^{21}=4(\operatorname{Pr} m)(\operatorname{Pr}) \int_{y_{e}}^{y_{e+1}}\left(\psi_{i}^{e}\right)\left(\frac{\partial \psi_{j}^{e}}{\partial y}\right) d y \\
& +4(\operatorname{Pr} m) \int_{y_{e}}^{y_{e+1}}\left(\frac{\partial \psi_{i}^{e}}{\partial y}\right)\left(\frac{\partial \psi_{j}^{e}}{\partial y}\right) d y \\
& K_{i j}^{22}=(\operatorname{Pr} m)(\operatorname{Pr})(R) \int_{y_{e}}^{y_{e+1}}\left(\psi_{i}^{e}\right)\left(\psi_{j}^{e}\right) d y, \\
& K_{i j}^{23}=-4(\operatorname{Pr} m)(E c)(\operatorname{Pr}) \int_{y_{e}}^{y_{e+1}}\left(\psi_{i}^{e}\right)\left(\frac{\partial \psi_{j}^{e}}{\partial y}\right)\left(\frac{\partial \psi_{j}^{e}}{\partial y}\right) d y \\
& -4(E c)(\operatorname{Pr}) \int_{y_{e}}^{y_{e+1}}\left(\psi_{i}^{e}\right)\left(\frac{\partial \psi_{i}^{e}}{\partial y}\right)\left(\frac{\partial \psi_{j}^{e}}{\partial y}\right) d y, \\
& K_{i j}^{31}=\int_{y_{e}}^{y_{e+1}}\left(\frac{\partial \psi_{i}^{e}}{\partial y}\right)\left(\frac{\partial \psi_{j}^{e}}{\partial y}\right) d y-M(\operatorname{Pr} m) \int_{y_{e}}^{y_{e+1}}\left(\psi_{i}^{e}\right)\left(\frac{\partial \psi_{j}^{e}}{\partial y}\right) d y, \\
& K_{i j}^{32}=-(\operatorname{Pr} m) \int_{y_{e}}^{y_{e+1}}\left(\psi_{i}^{e}\right)\left(\frac{\partial \psi_{j}^{e}}{\partial y}\right) d y, \\
& K_{i j}^{41}=\int_{y_{e}}^{y_{e+1}}\left(\frac{\partial \psi_{i}^{e}}{\partial y}\right)\left(\frac{\partial \psi_{j}^{e}}{\partial y}\right) d y-(S c) \int_{y_{e}}^{y_{e+1}}\left(\psi_{i}^{e}\right)\left(\frac{\partial \psi_{j}^{e}}{\partial y}\right) d y, \\
& K_{i j}^{42}=(K r)(S c) \int_{y_{e}}^{y_{e+1}}\left(\psi_{i}^{e}\right)\left(\frac{\partial \psi_{j}^{e}}{\partial y}\right) d y, b_{i}^{1 e}=\left[\left(\psi_{i}^{e}\right)\left(1+\frac{1}{\gamma}\right)\left(\frac{\partial \psi_{j}^{e}}{\partial y}\right)\right]_{y_{e}}^{y_{e+1}}, \\
& b_{i}^{2 e}=\left[4(\operatorname{Pr} m)\left(\psi_{i}^{e}\right)\left(\frac{\partial \psi_{j}^{e}}{\partial y}\right)\right]_{y_{e}}^{y_{e+1}}, b_{i}^{3 e}=\left[\left(\psi_{i}^{e}\right)\left(\frac{\partial \psi_{j}^{e}}{\partial y}\right)\right]_{y_{e}}^{y_{e+1}}, \\
& b_{i}^{4 e}=\left[\left(\psi_{i}^{e}\right)\left(\frac{\partial \psi_{j}^{e}}{\partial y}\right)\right]_{y_{e}}^{y_{e+1}}
\end{aligned}
$$

In one - dimensional space, linear element, quadratic element, or element of higher order can be taken. The whole domain is divided into a set of 90 intervals of equal length 0.1 . At each node 4 functions are to be evaluated. Hence after assembly of the elements we obtain a set of 364 equations which are nonlinear. Therefore, an iterative scheme must be utilized in the solution. After imposing the boundary conditions, a system of equations has been obtained which is solved by the Gauss elimination method while maintaining an accuracy of 0.00005. A convergence criterion based on the relative difference between the current and previous iterations is employed. When these differences satisfy the desired accuracy, the solution is assumed to have been converged and iterative process is terminated. The Gaussian quadrature is implemented for solving the integrations. The code of the algorithm has been executed in MATLAB running on a PC. Excellent convergence was achieved for all the results.

\section{STUDY OF GRID INDEPENDENCE OF FINITE ELEMENT METHOD}

In general, to study the grid independency/dependency, the mesh size should be varied in order to check the solution at different mesh (grid) sizes and get a range at which there is no variation in the solution. The numerical values of velocity $(u)$, Induced magnetic field $(B)$, temperature $(\theta)$ and concentration $(\phi)$ for different values of mesh (grid) size are shown in table 1. From this table, the authors observed that, there is no variation in the values of velocity $(u)$, Induced magnetic field $(B)$, temperature $(\theta)$ and concentration $(\phi)$ for different values of mesh (grid) size. Hence, it is concluded that the results are independent of mesh (grid) size.

Table-1. The numerical values of $u, B, \theta$ and $\phi$ for variation of mesh sizes

\begin{tabular}{|c|c|c|c|}
\hline \multicolumn{5}{|c|}{ Mesh (Grid) Size $=0.0001$} \\
\hline$u$ & $B$ & $\theta$ & $\phi$ \\
\hline 0.0000000 & 0.0000000 & 1.0000000 & 1.0000000 \\
\hline 8.4460812 & -0.2041255 & 0.5721116 & 0.4081449 \\
\hline 5.9442046 & -0.1491618 & 0.2910177 & 0.1737355 \\
\hline 3.5963027 & -0.0830346 & 0.1394854 & 0.0760272 \\
\hline 2.2666039 & -0.0416294 & 0.0645083 & 0.0337616 \\
\hline 1.5977422 & -0.0197623 & 0.0290949 & 0.0150684 \\
\hline 1.2759373 & -0.0090444 & 0.0128627 & 0.0067062 \\
\hline 1.1238416 & -0.0039951 & 0.0055456 & 0.0029605 \\
\hline 1.0519732 & -0.0016569 & 0.0022434 & 0.0012739 \\
\hline \multicolumn{5}{|c|}{ Mesh (Grid) Size $=0.001$} & \multicolumn{1}{|c|}{} \\
\hline$u$ & $B$ & $\theta$ & $\phi$ \\
\hline 0.0000000 & 0.0000000 & 1.0000000 & 1.0000000 \\
\hline 8.4462466 & -0.2040831 & 0.5722164 & 0.4081766 \\
\hline 5.9444105 & -0.1491182 & 0.2911392 & 0.1737549 \\
\hline 3.5965049 & -0.0829936 & 0.1395977 & 0.0760351 \\
\hline 2.2667601 & -0.0415915 & 0.0645761 & 0.0337687 \\
\hline 1.5978651 & -0.0197309 & 0.0291499 & 0.0150639 \\
\hline 1.2760032 & -0.0090361 & 0.0128930 & 0.0067006 \\
\hline 1.1238863 & -0.0039974 & 0.0055546 & 0.0029542 \\
\hline 1.0519907 & -0.0016433 & 0.0022529 & 0.0012715 \\
\hline \multicolumn{5}{|c|}{ Mesh (Grid) Size $=0.01$} \\
\hline$u$ & $B$ & $\theta$ & $\phi$ \\
\hline 0.0000000 & 0.0000000 & 1.0000000 & 1.00000000 \\
\hline 8.4464122 & -0.2040415 & 0.5723041 & 0.4081969 \\
\hline 5.9446244 & -0.1490713 & 0.2912565 & 0.1737774 \\
\hline 3.5966984 & -0.0829564 & 0.1396849 & 0.0760499 \\
\hline 2.2669106 & -0.0415682 & 0.0646439 & 0.0337636 \\
\hline 1.5979718 & -0.0197191 & 0.0291948 & 0.0150650 \\
\hline 1.2760849 & -0.0090278 & 0.0129119 & 0.0067019 \\
\hline 1.1239364 & -0.0039836 & 0.0055744 & 0.0029546 \\
\hline 1.0520188 & -0.0016424 & 0.0022639 & 0.0012772 \\
\hline
\end{tabular}

\section{CODE VALIDATION}

For program code validation, the author compared the present numerical results with analytical results of Prakash et al. (2014), Raptis et al. (2003) and Ahmed (2010) in tables 2, 3 and 4 respectively which are available in literature. From these tables, the author observed that the results are in good agreement with their study.

Table-2: Comparison between the present Velocity, Induced magnetic field and Temperature results with the results of Prakash et al. (2014) for Prm $<<M$.

\begin{tabular}{|c|c|c|c|c|c|c|}
\hline \multirow{2}{*}{$y$} & \multicolumn{2}{|c|}{ Results of Prakash et al. (2014) } & \multicolumn{3}{c|}{ Present numerical results } \\
\cline { 2 - 7 } & $u$ & $B$ & $\theta$ & $u$ & $B$ & $\theta$ \\
\cline { 2 - 7 } & $M=0.5$ & $M=0.5$ & $R=0.5$ & $M=0.5$ & $M=0.5$ & $R=0.5$ \\
\hline 0 & 0.0000 & 0.0000 & 1.0000 & 0.00000 & 0.00000 & 1.00000 \\
\hline 2 & 3.4360 & -0.2078 & 0.1961 & 3.43596 & -0.20782 & 0.19613 \\
\hline 4 & 1.8516 & -0.3093 & 0.0384 & 1.85163 & -0.30933 & 0.03841 \\
\hline 6 & 1.2335 & -0.2961 & 0.0075 & 1.23354 & -0.29610 & 0.00756 \\
\hline 8 & 1.0591 & -0.2537 & 0.0015 & 1.05917 & -0.25377 & 0.00150 \\
\hline 10 & 1.0146 & -0.2105 & 0.0003 & 1.01460 & -0.21059 & 0.00033 \\
\hline
\end{tabular}


Table-3: Comparison between the present velocity results with the velocity results of Prakash et al. (2014), Raptis et al. (2003) and Ahmed (2010) for Prm $=0.1<M=0.25, G r=5.0$, $\gamma=0.0, \psi=0.0$ and $G c=0.0$.

\begin{tabular}{|c|c|c|c|c|}
\hline$y$ & $\begin{array}{c}\text { Results of } \\
\text { Raptis et al. } \\
(2003)\end{array}$ & $\begin{array}{c}\text { Results of } \\
\text { Prakash et } \\
\text { al. (2014) }\end{array}$ & $\begin{array}{c}\text { Results of } \\
\text { Ahmed (2010) }\end{array}$ & $\begin{array}{c}\text { Present velocity } \\
\text { results }\end{array}$ \\
\cline { 2 - 5 } & $R=0.1$ & $R=0.1$ & $R=0.1$ & $R=0.1$ \\
\hline 0 & 0.00000 & 0.0000 & 0.00000 & 0.00000000 \\
\hline 2 & 3.39344 & 3.2584 & 3.35839 & 3.40218974 \\
\hline 4 & 1.88766 & 1.8523 & 1.85232 & 1.88624982 \\
\hline 6 & 1.24757 & 1.2406 & 1.24063 & 1.25430981 \\
\hline 8 & 1.06255 & 1.0613 & 1.06134 & 1.07639412 \\
\hline 10 & 1.01511 & 1.0149 & 1.01492 & 1.01569247 \\
\hline
\end{tabular}

Table-4: Comparison between the present Induced magnetic field results with the results of Prakash et al. (2014), Raptis et al. (2003) and Ahmed (2010) for Prm $=0.1<M=0.25, G r=5.0, \gamma=0.0, \psi=0.0$ and $G c=0.0$.

\begin{tabular}{|c|c|c|c|c|}
\hline \multirow{2}{*}{$y$} & $\begin{array}{c}\text { Results of } \\
\text { Raptis et al. } \\
(2003)\end{array}$ & $\begin{array}{c}\text { Results of } \\
\text { Prakash } \text { et } \\
\text { al. }(2014)\end{array}$ & $\begin{array}{c}\text { Results of } \\
\text { Ahmed (2010) }\end{array}$ & $\begin{array}{c}\text { Present Induced } \\
\text { Magnetic field } \\
\text { results }\end{array}$ \\
\cline { 2 - 5 } & $R=0.1$ & $R=0.1$ & $R=0.1$ & $R=0.1$ \\
\hline 0 & 0.00000 & 0.00000 & 0.00000 & 0.00000000 \\
\hline 2 & -0.09437 & -0.0875 & -0.08750 & -0.08842195 \\
\hline 4 & -0.14786 & -0.1388 & -0.13880 & -0.13995418 \\
\hline 6 & -0.14358 & -0.1354 & -0.13538 & -0.13695178 \\
\hline 8 & -0.12354 & -0.1167 & -0.11669 & -0.12615484 \\
\hline 10 & -0.10263 & -0.0970 & -0.09699 & -0.01245862 \\
\hline
\end{tabular}

\section{RESULTS AND DISCUSSION}

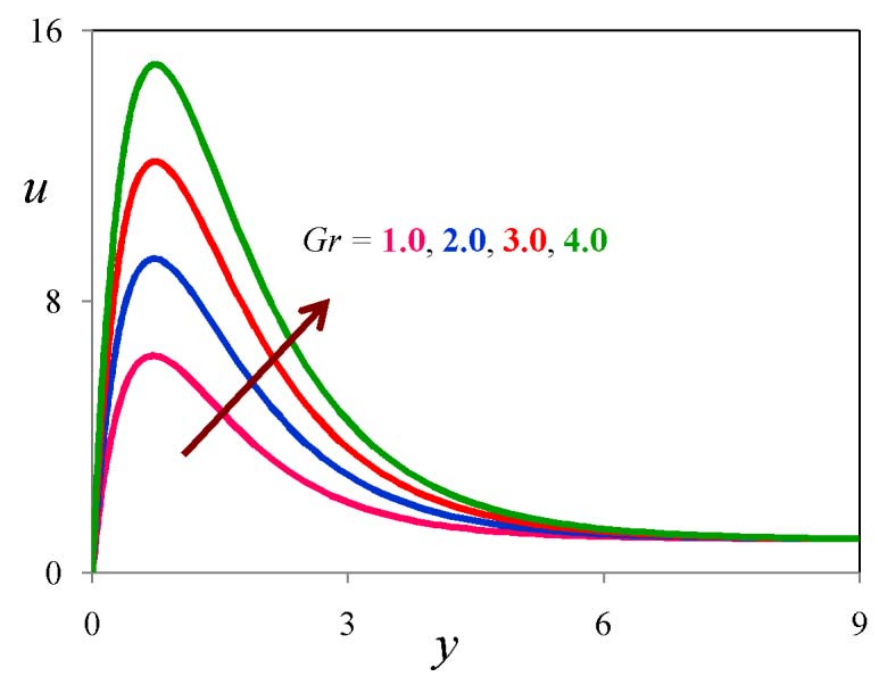

Fig. 2 Influence of $G r$ on velocity profiles

Numerical solutions of non-linear coupled partial differential equations (16)-(19) under boundary conditions (20) are obtained through finite element Galerkin technique for fluid velocity, temperature, concentration and induced magnetic field. The behaviour of the fluid (i. e., fluid velocity, temperature, induced magnetic field and concentration) was analyzed and also Skin-friction, Nusselt number and Sherwood number with various physical parameters at $y=1.0$ through the Figs. (2)-(20). In this study the boundary condition for $y \rightarrow \infty$ is replaced by $y_{\max }=9$ is a sufficiently large value of $y$, where the velocity, induced magnetic field, temperature and concentration profiles can be approached to the relevant free stream velocity. The variation of numerical values of thermal Grashof number or Grashof number for heat transfer $(\mathrm{Gr})$ on velocity profiles at the boundary layer is as shown in the Fig. 2. It is observed that an increase in $G r$ leads to increase in the fluid velocity due to enhancement in buoyancy force. Here, the positive values of $G r$ correspond to cooling of the plate. In addition, it is observed that the velocity increases sharply near the wall as $G r$ increases and then decays to the free stream value.

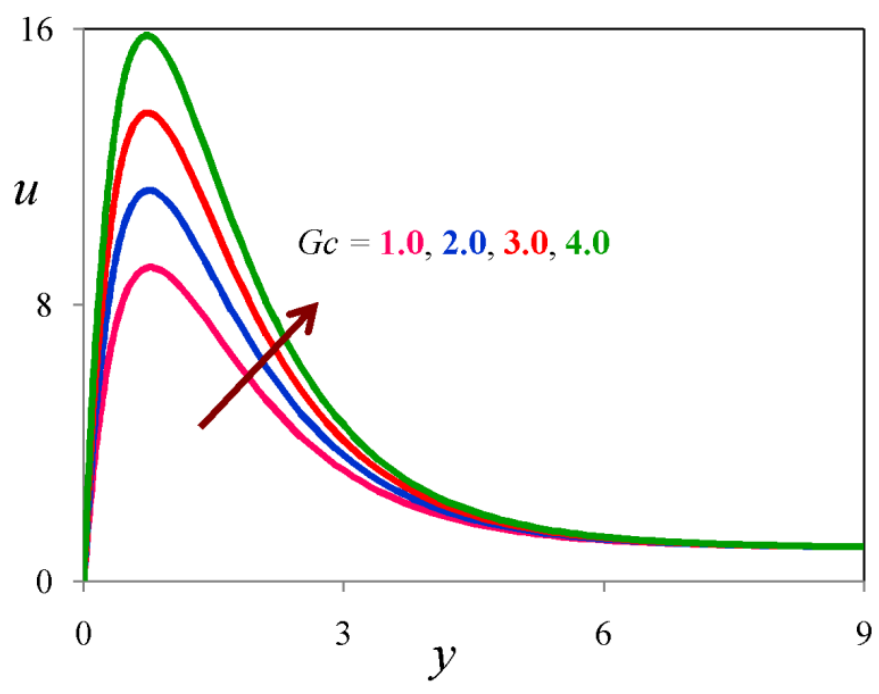

Fig. 3 Influence of $G c$ on velocity profiles

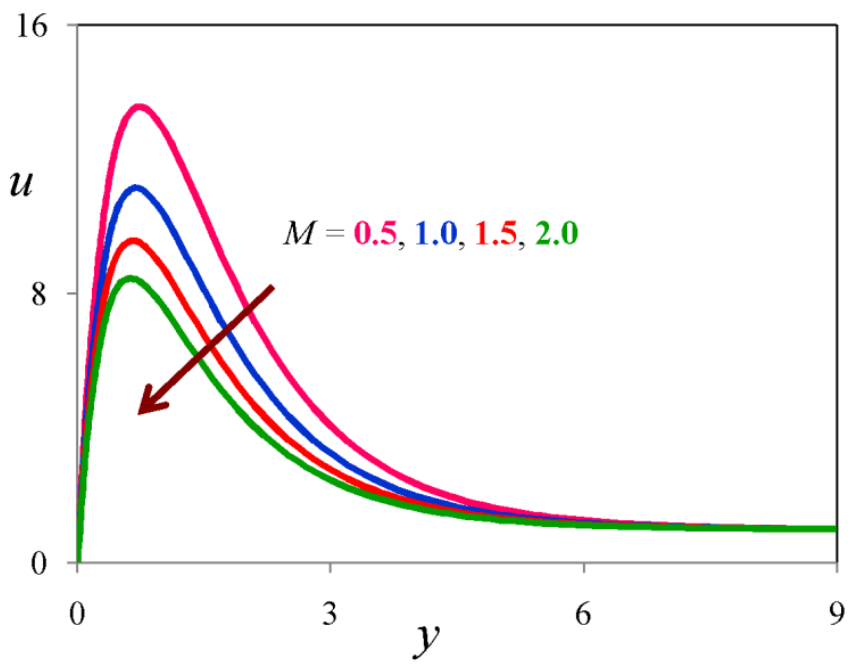

Fig. 4 Influence of $M$ on velocity profiles

Fig. 3 shows the influence of Grashof number for mass transfer $(G c)$ on the velocity profile at the boundary layer. The velocity distribution attains a distinctive maximum value in the vicinity of the plate and then decreases properly to approach a free stream value. As expected, the fluid velocity increases and the peak value becomes more distinctive due to increase in the buoyancy force represented by $G c$. The effect of the Hartmann number (or) Magnetic field parameter is shown in Fig. 4. It is observed that the velocity of the fluid decreases with the increase of the magnetic field parameter values. The decrease in the velocity as the Magnetic field parameter increases is because the presence of a magnetic field in an electrically conducting fluid introduces a force called the Lorentz force, which acts against the flow if the magnetic field is applied in the normal direction, as in the present study. This resistive force slows down the fluid velocity component. Figs. 5 and 6 
show the behaviour of fluid velocity and fluid temperature respectively with an influence of Prandtl number. Fluid velocity and temperature decreases as increasing of Prandtl number due to enhancement of the thermal conductivity. Since Prandtl number is the ratio of momentum diffusivity to thermal conductivity. For small Prandtl number, thermal conductivity is high and momentum diffusivity is low, because of that fluid viscosity is low. Therefore reduce the heat transfer for small Prandtl number.

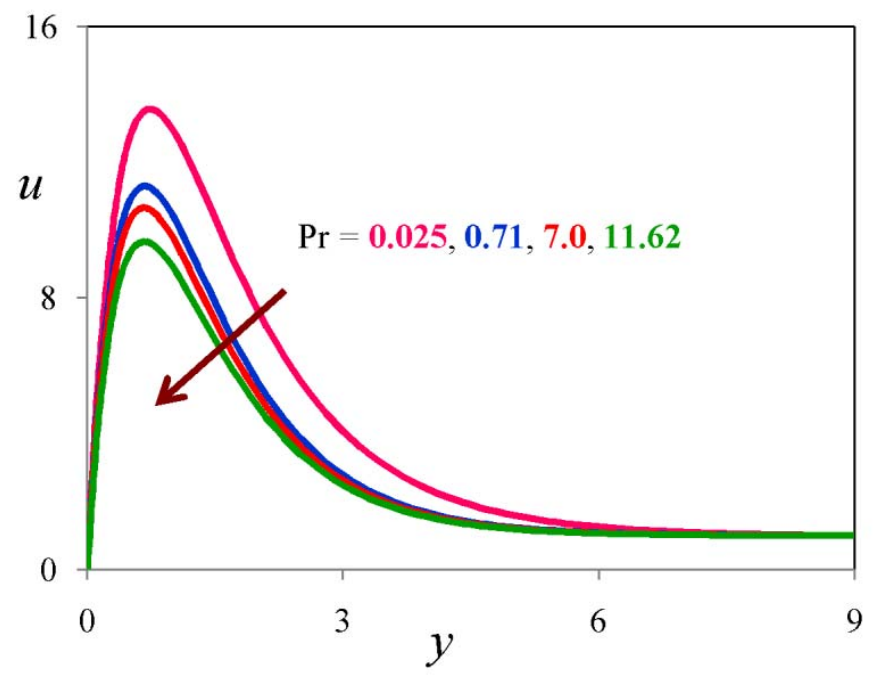

Fig. 5 Influence of Pr on velocity profiles

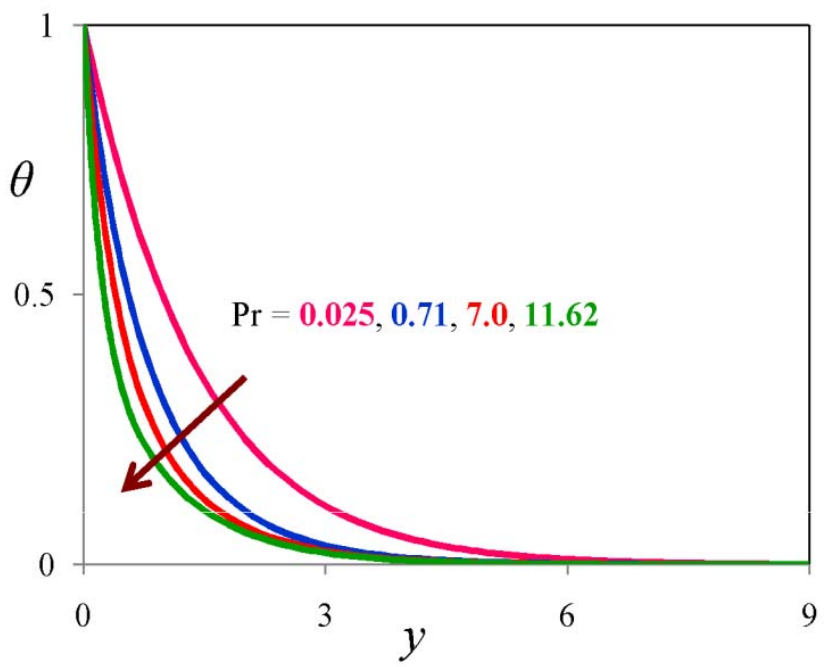

Fig. 6 Influence of Pr on temperature profiles

Figures 7 and 8 show that, influence of Schmidt number $(S c)$ on velocity profile and temperature profile. It is observed that, Schmidt number $(S c)$ is in the concentration and it is coupled in the momentum equation. Increasing of Schmidt number $(S c)$ the momentum boundary layer thickness is increasing as well as fluid velocity and fluid concentration is decreasing in the entire boundary of the region. The effect of the viscous dissipation parameter i.e., the Eckert number $E c$ on the velocity and temperature are shown in Figs. 9 and 10 respectively. The Eckert number $E c$ expresses the relationship between the kinetic energy in the flow and the enthalpy. It embodies the conversion of kinetic energy into internal energy by work done against the viscous fluid stresses. Greater viscous dissipative heat causes a rise in the temperature, as well as the velocity and cross flow velocity. This behaviour is evident from Figs. 9 and 10.

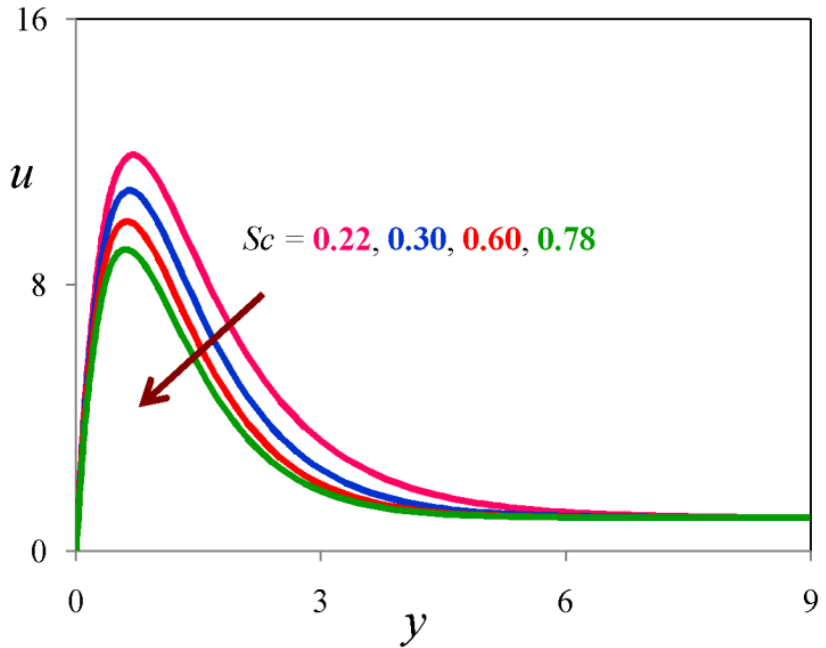

Fig. 7 Influence of $S c$ on velocity profiles

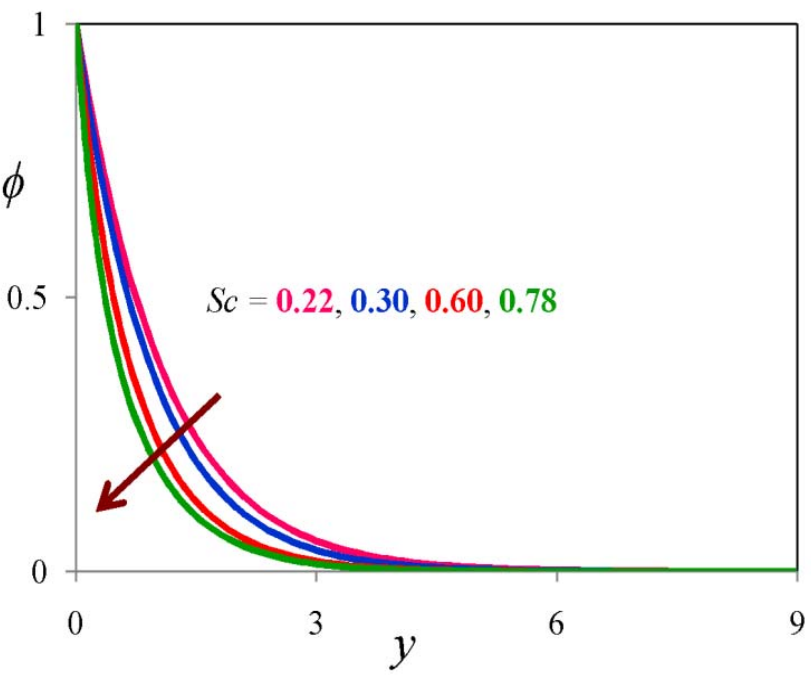

Fig. 8 Influence of $S c$ on concentration profiles

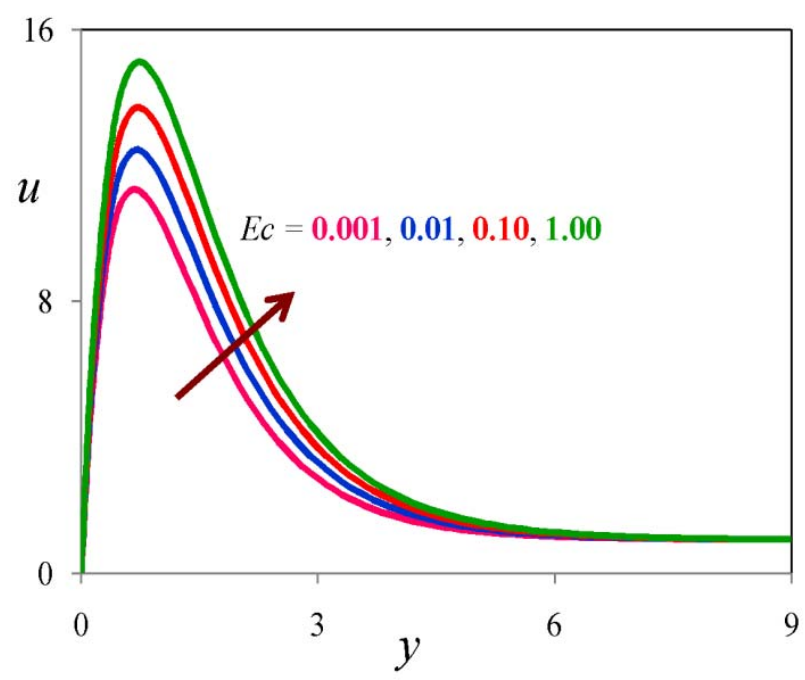

Fig. 9 Influence of $E c$ on velocity profiles

Figs. 11 and 12 show the influence of magnetic Prandtl number Prm on the velocity of fluid and temperature of fluid. Both fluid velocity and temperature decrease as increasing of magnetic Prandt number Prm. The effects of the thermal radiation parameter $R$ on the velocity and temperature profiles in the boundary layer are illustrated in 
Figs. 13 and 14 respectively. Increasing the thermal radiation parameter produces significant increase in the thermal condition of the fluid and its thermal boundary layer. This increase in the fluid temperature induces more flow in the boundary layer causing the velocity of the fluid there to increase. As expected, the presence of the chemical reaction significantly affects the concentration profiles as well as the velocity profiles from Figs. 15 and 16. It should be mentioned that the studied case is both for destructive and generative chemical reaction. In fact, as chemical reaction increases, the considerable reduction in the velocity profiles is predicted, and the presence of the peak indicates that the maximum value of the velocity occurs in the body of the fluid close to the surface but not at the surface. Also, with an increase in the chemical reaction parameter, the concentration decreases. It is evident that the increase in the chemical reaction significantly alters the concentration boundary layer thickness but does not alter the momentum boundary layers.

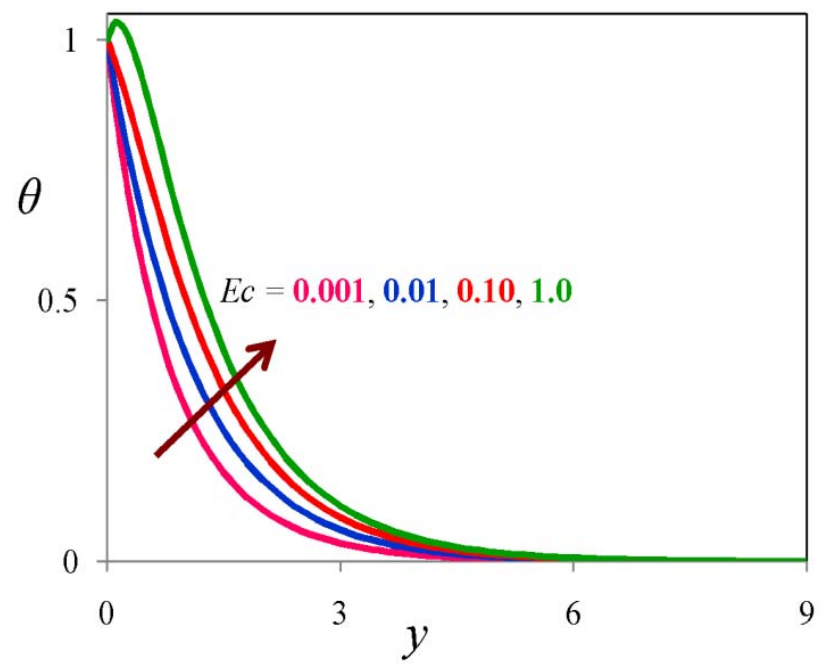

Fig. 10 Influence of $E c$ on temperature profiles

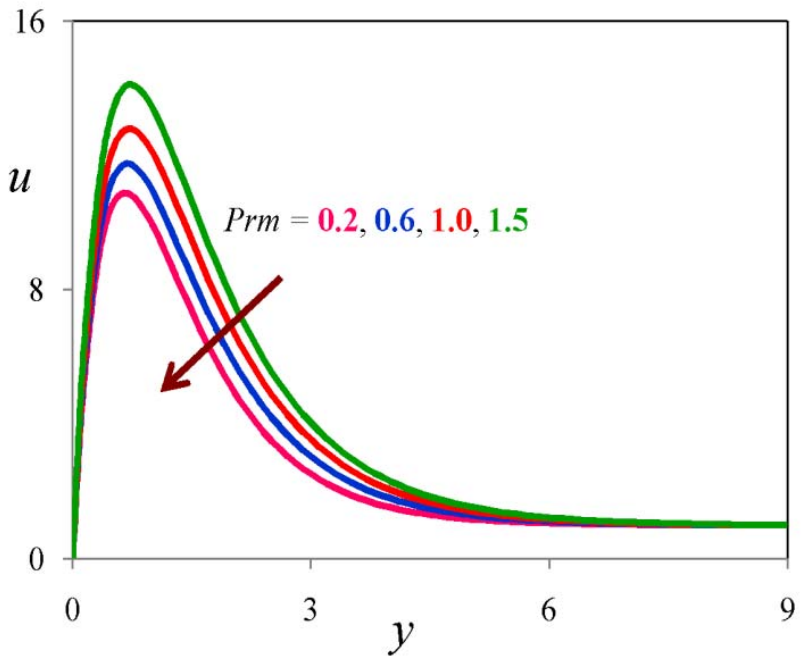

Fig. 11 Influence of Prm on velocity profiles

Fig. 17 shows the fluid velocity with an influence of Casson fluid parameter. With an increasing Casson parameter, the fluid velocity decreases in the entire boundary region. Since fluid viscosity will be high with increase of Casson parameter, momentum boundary layer thickness decreases. An influence of angle of inclination of the plate on the velocity profile is shown in the Fig. 18. It is observed that fluid velocity decreases with increasing inclination parameter $(\psi)$. The response of induced magnetic field to Hartmann number is shown in the
Fig. 19 for weak buoyancy and airflow. Clearly, as Hartmann number increases, the induced magnetic field reduces. The effect of magnetic Prandtl number Prm on the induced magnetic field is presented in the Fig. 20. In this figure magnetic Prandtl number Prm is set as less than unity, which implies that the magnetic diffusion rate exceeds the viscous diffusion rate. As such Prm increases, momentum diffusivity will increase. Therefore, Prm increases from 0.2 to 1.5 , the induced magnetic field is found to increase absolutely in the boundary layer $0 \leq y \leq 6$, but this trend is opposite for the region $6 \leq y \leq 9$. Greater flux reversal arises in the boundary layer region $y \in[0,6]$ and for Prm $=0.2$ (magnetic diffusion rate exceeds the viscous diffusion rate); but this trend is reversed for the region $y \in[6,9]$.

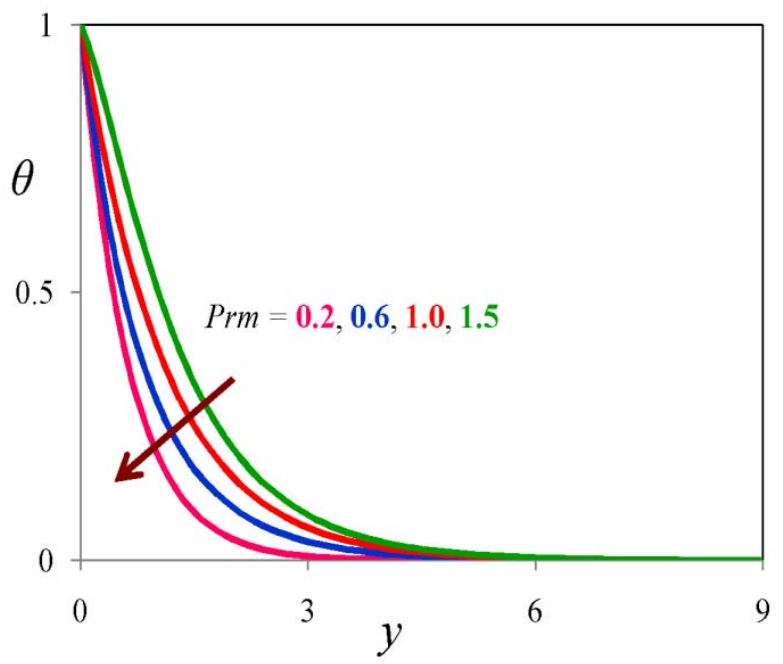

Fig. 12 Influence of Prm on temperature profiles

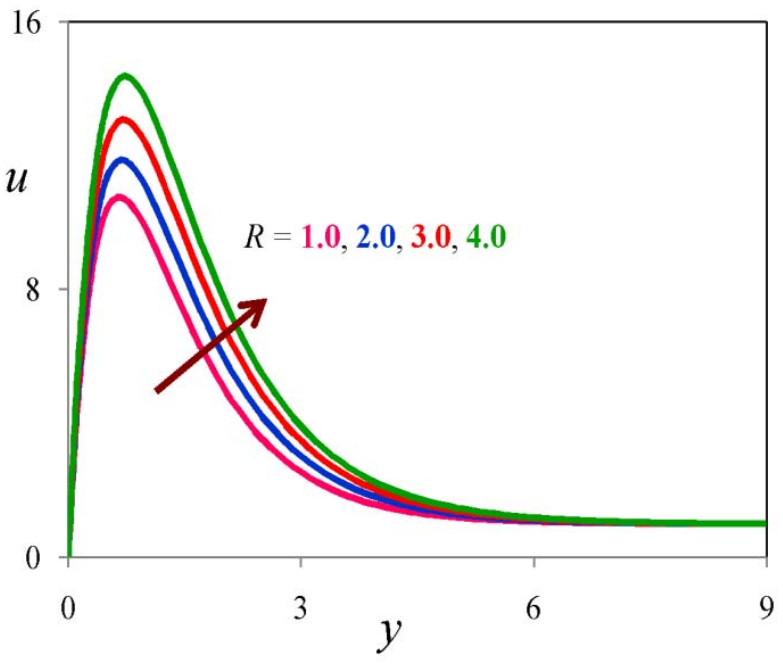

Fig. 13 Influence of $R$ on velocity profiles

Tables 5 and 6 represent the variations of Ec, R, $\gamma$ and Prm, Kr, $\psi$ on skin-friction coefficient respectively. The skin-friction coefficient is increasing with increasing values of $E c$ and decreasing with increasing values of $R$ and $\gamma$. The influences of $R, \operatorname{Pr}$ and $E c$ on rate of heat transfer coefficient or Nusselt number are discussed in table 7 with the help of numerical values. The rate of heat transfer coefficient is increasing with increasing values of $E c$ and the reverse effect is observed with increasing values of $R$ and Pr. The combined influence of $S c$ and $K r$ on rate of mass transfer coefficient or Sherwood number is discussed in table 8. From this table, the authors observed that the rate of mass transfer coefficient is decreasing with increasing values of $S c$ and $K r$. 


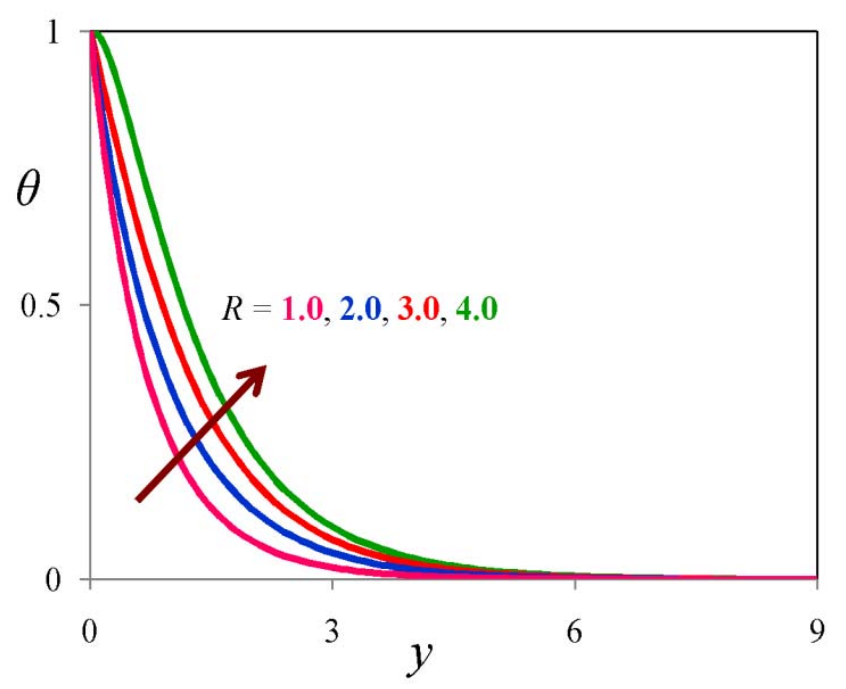

Fig. 14 Influence of $R$ on temperature profiles

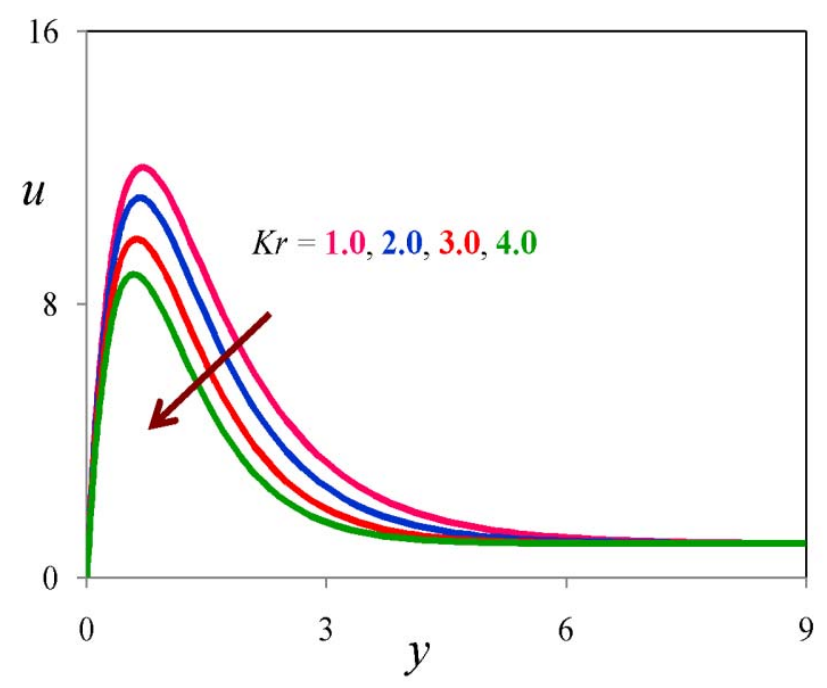

Fig. 15 Influence of $K r$ on velocity profiles

Table-5: Skin-friction values for variation of $E c, R$ and $\gamma$

\begin{tabular}{|c|c|c|c|}
\hline$E c$ & $R$ & $\gamma$ & $C_{f}$ \\
\hline 0.001 & 0.1 & 0.5 & 3.26154832 \\
\hline 1.000 & 0.1 & 0.5 & 3.28015548 \\
\hline 0.001 & 0.5 & 0.5 & 3.27336541 \\
\hline 0.001 & 0.1 & 1.0 & 3.20667124 \\
\hline
\end{tabular}

Table-6: Skin-friction values for variation of $\mathrm{Prm}, \mathrm{Kr}$ and $\psi$

\begin{tabular}{|c|c|c|c|}
\hline Prm & $K r$ & $\psi$ & $C_{f}$ \\
\hline 0.2 & 1.0 & $30^{\circ}$ & 3.26154832 \\
\hline 0.6 & 1.0 & $30^{\circ}$ & 3.19224862 \\
\hline 0.2 & 2.0 & $30^{\circ}$ & 3.21448012 \\
\hline 0.2 & 1.0 & $45^{\circ}$ & 3.22466215 \\
\hline
\end{tabular}

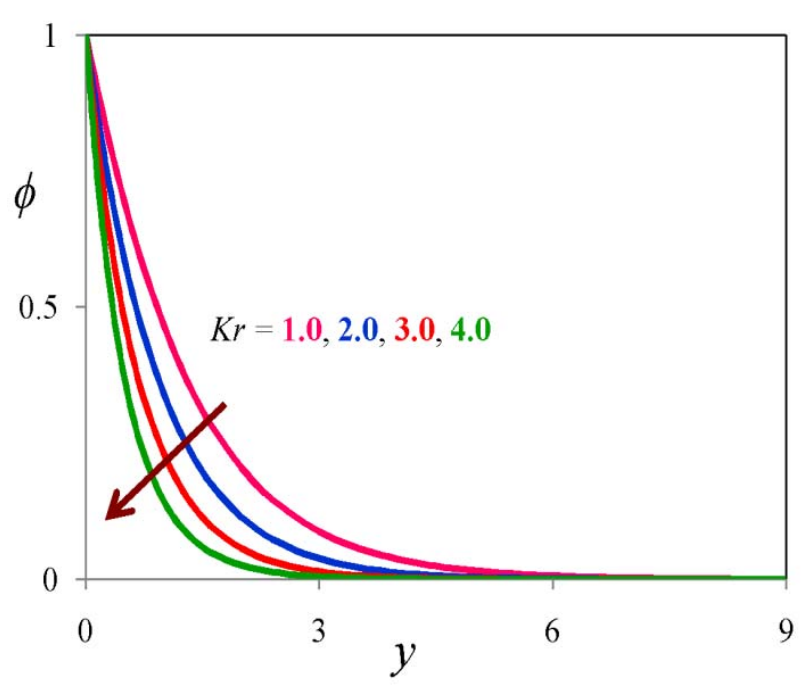

Fig. 16 Influence of $\mathrm{Kr}$ on concentration profiles

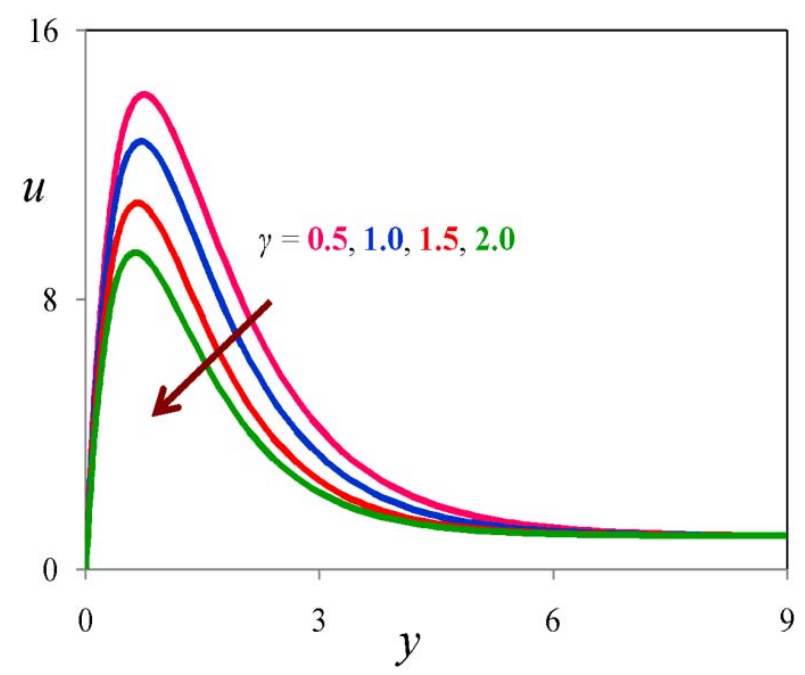

Fig. 17 Influence of $\gamma$ on velocity profiles

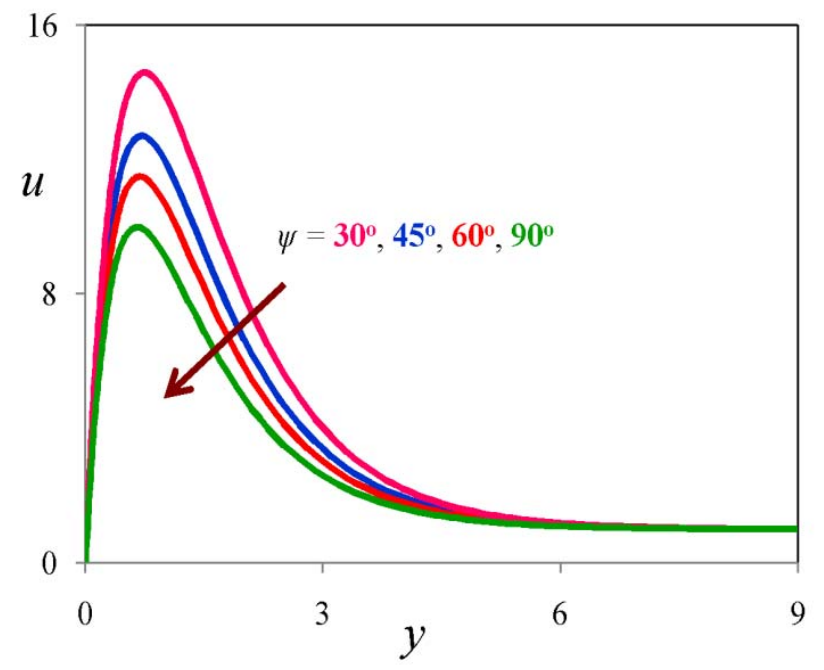

Fig. 18 Influence of $\psi$ on velocity profiles 


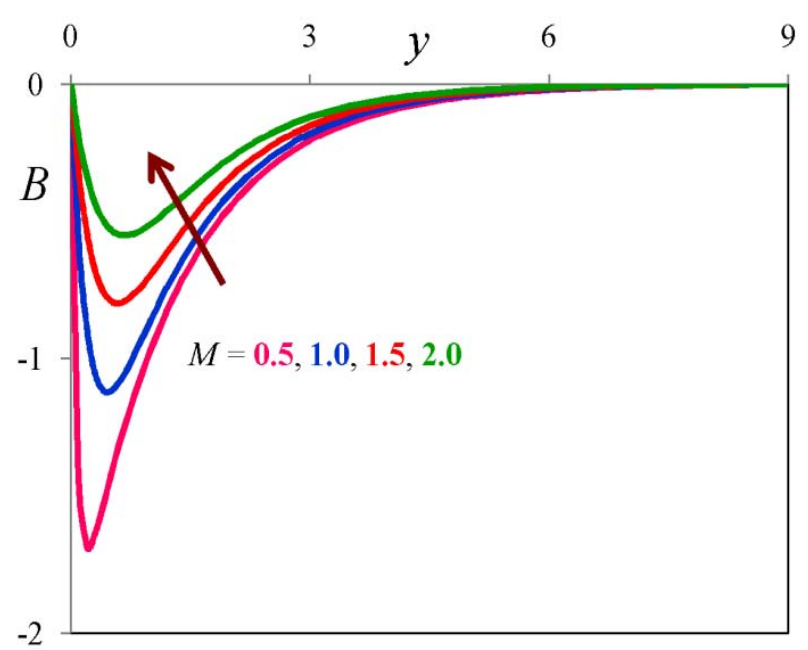

Fig. 19 Influence of $M$ on induced magnetic field

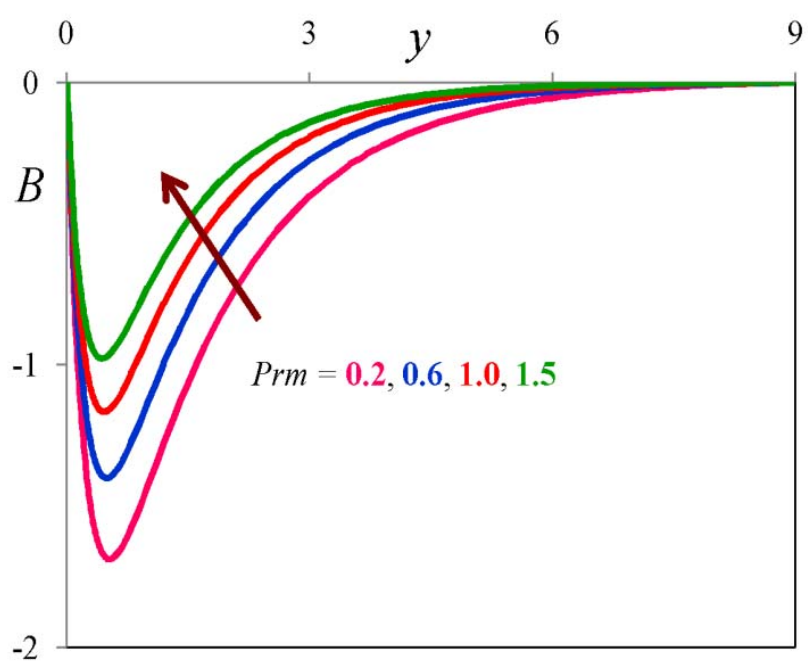

Fig. 20 Influence of Prm on induced magnetic field

Table-7: Nusselt number values for variation of $E c, R$ and $\mathrm{Pr}$

\begin{tabular}{|c|c|c|c|}
\hline$E c$ & $R$ & $\operatorname{Pr}$ & $N u$ \\
\hline 0.001 & 0.1 & 0.71 & 0.62334821 \\
\hline 1.000 & 0.1 & 0.71 & 0.63955478 \\
\hline 0.001 & 0.5 & 0.71 & 0.64388721 \\
\hline 0.001 & 0.1 & 7.00 & 0.57661548 \\
\hline
\end{tabular}

Table-8: Sherwood number values for variation of $S c$ and $K r$

\begin{tabular}{|c|c|c|}
\hline$S c$ & $K r$ & $S h$ \\
\hline 0.2 & 1.0 & 0.59348752 \\
\hline 0.6 & 1.0 & 0.56177823 \\
\hline 0.2 & 2.0 & 0.55395842 \\
\hline
\end{tabular}

\section{CONCLUSIONS}

The present study addressed the characteristics of heat and mass transfer on unsteady magnetohydrodynamic free convective Casson fluid flow of an optically thick fluid over an inclined vertical plate with magnetic and viscous dissipations. The set of fundamental governing equations have been solved numerically using finite element method.

The numerical solutions have been developed for velocity, induced magnetic field, temperature, concentration, Skin-friction, Rate of heat and mass transfer coefficients. The features of the flow characteristics were analyzed by plotting graphs and discussed in detail.

- The influence of Grashof number for heat and mass transfer stabilizes the momentum boundary layer growth.

- Increasing the values of Casson fluid parameter, Hartmann number and Angle of inclination of the plate retards the velocity of the flow field at all points.

- A growing Prandtl number decreases temperature of the flow field at all points and increases with increasing thermal radiation and viscous dissipation parameters.

- Induced Magnetic field decreases with the increasing Hartmann number.

- Magnetic Prandtl number deeply influence on the induced magnetic field in the thermal boundary layer i.e. induced magnetic field decreases with the increase in the Magnetic Prandtl number.

- The concentration reduces with rising of Schmidt number.

- The velocity as well as concentration reduces with an enlargement in the chemical reaction parameter.

- The numerical results are obtained and compared with formerly reported cases available in the open literature and they are found to be in very good concurrence.

The present study has been confined to non-newtonian viscous model. Future investigations will consider viscoelastic and power-law rheological fluid models and will be communicated in the near future.

\section{ACKNOWLEDGEMENT}

Authors are grateful to the reviewers for their valuable comments and suggestions which helped them to improve the quality of this research paper. Also the authors are thankful to Dr. K. Tejaswani, GITAM University, Hyderabad campus, for her inputs.

\section{NOMENCLATURE}

$C_{\infty}^{\prime} \quad$ Concentration of the fluid far away from the plate $\left(\mathrm{Kg} \mathrm{m}^{-3}\right)$

$C_{w}^{\prime}$

$y$

$T_{\infty}^{\prime}$

Gc

$u$

Sh

$v^{\prime}$

$u^{\prime}$

$g$

$a$

$K r$

$x^{\prime}$

$y^{\prime}$

$\bar{J}$

$k_{r}^{\prime}$

Ec

$C^{\prime}$

$T^{\prime}$

$T_{w}^{\prime}$
Concentration of the plate $\left(\mathrm{Kg} \mathrm{m}^{-3}\right)$

Dimensionless displacement $(m)$

Fluid temperature away from the plate $(K)$

Grashof number for mass transfer

Non-dimensional fluid velocity $\left(m s^{-1}\right.$ )

The local Sherwood number

Velocity component $\left(m s^{-1}\right)$

Velocity component in $x^{\prime}-\operatorname{direction}\left(m s^{-1}\right)$

Acceleration of gravity, $9.81\left(\mathrm{~m} \mathrm{~s}^{-2}\right)$

A Constant

Chemical reaction parameter

Coordinate axis along the plate $(m)$

Co-ordinate axis normal to the plate $(m)$

Current density vector (A.s. $\mathrm{m}^{-2}$ )

Dimensional Chemical reaction parameter

Eckert number (Viscous Dissipation parameter)

Fluid Concentration $\left(\mathrm{Kg} \mathrm{m}^{-3}\right)$

Fluid Temperature $(K)$

Fluid temperature at the wall $(K)$ 


$\begin{array}{ll}G r & \text { Grashof number for heat transfer } \\ B & \text { Induced Magnetic field (Tesla) } \\ H_{x}^{\prime} & \begin{array}{l}\text { Induced Magnetic field along } x^{\prime}-\text { direction } \\ (\text { Tesla })\end{array} \\ H_{y}^{\prime} & \begin{array}{l}\text { Induced Magnetic field along } y^{\prime}-\text { direction } \\ \text { (Tesla) }\end{array} \\ M & \text { Magnetic field parameter (or) Hartmann number } \\ \operatorname{Pr} m & \text { Magnetic Prandtl number } \\ \operatorname{Pr} & \text { Prandtl number } \\ q_{r} & \text { Radiative heat transfer coefficient } \\ U_{o} & \text { Reference velocity at the plate }\left(m^{-1}\right) \\ \operatorname{Re} & \text { Reynolds number } \\ \left(J_{x}, J_{y}, J_{z}\right) & \text { Scalar Components of } \bar{J} \\ S c & \text { Schmidt number } \\ D & \text { Solute mass diffusivity }\left(m^{2} s^{-1}\right) \\ C_{p} & \text { Specific heat at constant pressure }\left(\mathrm{J} \mathrm{Kg}^{-1} \mathrm{~K}\right) \\ N u & \text { The local Nusselt number } \\ C_{f} & \text { The local skin-friction }\left(N m^{-2}\right) \\ B_{0} & \text { Uniform magnetic field }(T e s l a) \\ G\end{array}$

Greek Symbols: $\kappa$

$\theta$

$\tau_{w}^{\prime}$

$\beta^{*}$

\section{$\psi$}

$\gamma$

$\sigma$

v

$\mu_{e}$

$\phi$

$\sigma^{*}$

$\rho$

$\beta$

\section{Superscripts:}

/
Subscripts:
$\infty$
$p$
$w$

Thermal conductivity of the fluid ( $W m^{-1} K^{-1}$ )

Non dimensional fluid temperature $(K)$

Shear stress $\left(N \mathrm{~m}^{-2}\right)$

Volumetric Coefficient of thermal expansion with concentration $\left(\mathrm{m}^{3} \mathrm{Kg}^{-1}\right)$

Angle of inclination of plate ( degrees )

Casson fluid parameter

Electric conductivity of the fluid $\left(s \mathrm{~m}^{-1}\right)$

Kinematic viscosity $\left(m^{2} s^{-1}\right)$

Magnetic Permeability ( N. $A^{-2}$ )

Species concentration $\left(K g \mathrm{~m}^{-3}\right)$

Stefan Boltzmann Constant (W.m. $\left.K^{-4}\right)$

The constant density $\left(\mathrm{Kg} \mathrm{m}^{-3}\right)$

Volumetric coefficient of thermal expansion $\left(K^{-1}\right)$

Dimensionless Properties

Free stream conditions

Plate

Conditions on the wall

\section{REFERENCES}

Ahmed, S., 2010, "Induced Magnetic Field with Radiating Fluid over a Porous Vertical Plate: Analytical Study," J. Navel of Arch. Marine Eng., 7, 61-72.
Ahmed, S., and Mahdy, A., 2016, "Unsteady MHD Double Diffusive Convection in the Stagnation Region of an Impulsively Rotating Sphere in the Presence of Thermal Radiation Effect," J. Taiwan Inst. Chem. Eng., 58, 173-180. http://doi.org/10.1016/j.jtice.2015.06.033

Akbar, N.S., Nadeem, S., Haq, R.U., and Khan, Z.H., 2013, "Radiation Effects on MHD Stagnation Point Flow of Nanofluid Towards a Stretching Surface with Convective Boundary Condition," Chin. J. Aeronaut., 26, 1389-1397.

http://doi.org/10.1016/j.cja.2013.10.008

Bathe, K.J., 1996, "Finite Element Procedures," Prentice-Hall, New Jersey.

Bhargava, R., and Rana, P., 2011, "Finite Element Solution to Mixed Convection in MHD Flow of Micropolar Fluid along a Moving Vertical Cylinder with Variable Conductivity," Int. J. Appl. Math. Mech., 7, 29-51.

Bhattacharyya, K., 2013, "Boundary Layer Stagnation-Point Flow of Casson Fluid and Heat Transfer Towards a Shrinking/Stretching Sheet," Front. Heat Mass Transf., 4, 023003. http://dx.doi.org/10.5098/hmt.v4.2.3003.

Bhattacharyya, K., 2013, "MHD Stagnation-Point Flow of Casson Fluid and Heat Transfer over a Stretching Sheet with Thermal Radiation," $J$. Thermodyn., 2013, 169674.

http://dx.doi.org/10.1155/2013/169674

Bhattacharyya, K., Mukhopadhyay, S., Layek, G.C., and Pop, I., 2012, "Effects of Thermal Radiation on Micropolar Fluid Flow and Heat Transfer over a Porous Shrinking Sheet," Int. J. Heat Mass Transf., 55, 2945-2952.

http://doi.org/10.1016/j.ijheatmasstransfer.2012.01.051

Casson, M., 1959, "A Flow Equation for Pigment-Oil Suspensions of the Printing Ink Type,". In: C. C. Mills (ed.), Rheology of disperse systems, Newyork, Oxford: Pergamon. 84-104.

Chamkha, A.J., and Camille, I., 2000, "Effects of Heat Generation/Absorption and Thermophoresis on Hydromagnetic Flow with Heat and Mass Transfer over a Flat Surface," Int. J. Numer. Methods Heat Fluid Flow, 10, 432-448. http://dx.doi.org/10.1108/09615530010327404

Dash, R.K., Mehta, K.N., and Jayaraman, G., 1996, "Casson Fluid Flow in a Pipe Filled with a Homogeneous Porous Medium," Int. J. Eng. Sci., 34, 1145-1156.

https://doi.org/10.1016/0020-7225(96)00012-2

Dettmer, W., and Peric, D., 2006, "A Computational Framework for Fluid-Rigid Body Interaction: Finite Element Formulation and Applications," Comput. Methods Appl. Mech. Eng., 195, 1633-1666. http://doi.org/10.1016/j.cma.2005.05.033

Hansbo, A., and Hansbo, P., 2004, "A Finite Element Method for the Simulation of Strong and Weak Discontinuities in Solid Mechanics," Comput. Methods Appl. Mech. Eng., 193, 3523-3540.

http://doi.org/10.1016/j.cma.2003.12.041

Harinath Reddy, S., Raju, M. C., and Keshava Reddy, E., 2016, "Radiation Absorption and Chemical Reaction Effects on MHD Flow of Heat Generating Casson Fluid Past Oscillating Vertical Porous Plate," Front. Heat Mass Transf., 7, 21. http://dx.doi.org/10.5098/hmt.7.21 
Khalid, A., Khan, I., Khan, A., and Shafie, S., 2015, "Unsteady MHD Free Convection Flow of Casson Fluid Past over an Oscillating Vertical Plate Embedded in a Porous Medium," Eng. Sci. Technol. Int. J., 80, $1-9$.

http://doi.org/10.1016/j.jestch.2014.12.006

Lin, Y-Y., and Lo, S-P., 2003, "Finite Element Modeling for Chemical Mechanical Polishing Process under Different Back Pressures," J. Mater. Processing Technol., 140, 646-652.

http://doi.org/10.1016/S0924-0136(03)00767-2

Mukhopadhyay, S., Bhattacharyya, K., and Layek, G.C., 2011, "Steady Boundary Layer Flow and Heat Transfer over a Porous Moving Plate in Presence of Thermal Radiation," Int. J. Heat Mass Transf., 54, 2751-2757.

http://doi.org/10.1016/j.ijheatmasstransfer.2011.03.017

Mustafa, M., and Khan, J.A., 2015, "Model for Low of Casson Nanofluid Past a Nonlinearly Stretching Sheet Considering Magnetic Field Effects," AIP Adv., 5, 077148.

http://dx.doi.org/10.1063/1.4927449.

Nadeem, S., Haq, R.U., Akbar, N.S., and Khan, Z.H., 2013, "MHD Three-Dimensional Casson Fluid Flow Past a Porous Linearly Stretching Sheet," Alexandria Eng. J., 52, 577-582.

http://doi.org/10.1016/j.aej.2013.08.005

Nadeem, S., Mehmood, R., and Akbar, N.S., 2014, "Oblique Stagnation Point Flow of a Casson Nanofluid Towards a Stretching Surface with Heat Transfer," J. Comp. Theor. Nanosci., 11, 1422-1432.

https://doi.org/10.1166/jctn.2014.3513

Pal, D., Mandal, G., and Vajravelu, K., 2013, "MHD ConvectionDissipation Heat Transfer over a Non-Linear Stretching and Shrinking Sheets in Nanofluids with Thermal Radiation," Int. J. Heat Mass Transf., 65, 481-490.

http://doi.org/10.1016/j.ijheatmasstransfer.2013.06.017

Prakash, J., Vijaya Kumar, A.G., Rushi Kumar, B., and Varma, S.V.K., 2014, "The Effects of Induced Magnetic Field and Radiation on MHD Mixed Convective Flow over a Porous Vertical Plate in the Presence of Viscous and Magnetic Dissipations," Int. Conference on Adv. Marine, Industrial and Mech. Eng., 52-61.

Ramamohan Reddy, L., Raju, M. C., Raju, G.S.S., and Reddy, N.A., 2016, "Thermal Diffusion and Rotational Effects on Magnetohydrodynamic Mixed Convection Flow of Heat Absorbing/Generating Visco-Elastic Fluid Through a Porous Channel," Front. Heat Mass Transf., 7, 20.

http://dx.doi.org/10.5098/hmt.7.20

Raptis, A.A., Perdikis, C., and Leontitsis, A., 2003, "Effects of Radiation in an Optically Thin Gray Gas Flowing Past a Vertical Infinite Plate in the Presence of a Magnetic Field," Heat Mass Transf., 39, 771-773.

http://doi.org/10.1007/s00231-002-0317-8

Rashidi, M.M., Ali, M., Freidoonimehr, N., Rostami, B., and Hossain, M.A., 2014, "Mixed Convective Heat Transfer for MHD Viscoelastic
Fluid Flow over a Porous Wedge with Thermal Radiation," Adv. Mech. Eng.,

http://dx.doi.org/10.1155/2014/735939.

Reddy, J.N., 1985, "An Introduction to the Finite Element Method," McGraw - Hill, New York.

Sharada, K., and Shankar, B., 2015, "MHD Mixed Convection Flow of a Casson Fluid over an Exponentially Stretching Surface with the Effects of Soret, Dufour, Thermal Radiation and Chemical Reaction," World J. Mech., 5, 165-177.

http://dx.doi.org/10.4236/wjm

Siva Reddy Sheri, and Srinivasa Raju, R., 2015, "Soret Effect on Unsteady MHD Free Convective Flow Past a Semi-Infinite Vertical Plate in the Presence of Viscous Dissipation," Int. J. Comput. Methods Eng. Sci. Mech., 16, 132-141.

http://dx.doi.org/10.1080/15502287.2015.1009583

Siva Reddy Sheri, and Srinivasa Raju, R., 2016, "Transient MHD Free Convective Flow Past an Infinite Vertical Plate Embedded in a Porous Medium with Viscous Dissipation," Meccanica, 51, 1057-1068.

http://doi.org/10.1007/s11012-015-0285-y

Sivaiah, S., and Srinivasa Raju, R., 2013, "Finite Element Solution of Heat and Mass Transfer Flow with Hall Current, Heat Source and Viscous Dissipation," Appl. Math. Mech. English Edition, 34, 559-570. http://doi.org/10.1007/s10483-013-1690-8

Srinivasa Raju, R., Jithender Reddy, G., Anand Rao, J., Rashidi, M.M., and Gorla, R.S.R., 2016, "Analytical and Numerical Study of Unsteady MHD Free Convection Flow over an Exponentially Moving Vertical Plate with Heat Absorption," Int. J. Thermal Sci., 107, 303-315. http://doi.org/10.1016/j.ijthermalsci.2016.04.005

Srinivasa Raju, R., Mahesh Reddy, B., Rashidi, M.M., and Gorla, R.S.R., 2016, "Application of Finite Element Method to Unsteady MHD Free Convection Flow Past a Vertically Inclined Porous Plate Including Thermal Diffusion and Diffusion Thermo Effects," J. Porous Media, 19, 701-722.

http://doi.org/10.1615/JPorMedia.v19.i8.40

Su, X., Zheng, L., Zhang, X., and Zhang, J., 2012, "MHD Mixed Convective Heat Transfer over a Permeable Stretching Wedge with Thermal Radiation and Ohmic Heating," Chem. Eng. Sci., 78, 1-8. http://doi.org/10.1016/j.ces.2012.04.026

Venkateswarlu, B., and Satya Narayana, P.V., 2016, "Influence of Variable Thermal Conductivity on MHD Casson Fluid Flow over a Stretching Sheet with Viscous Dissipation, Soret and Dufour Effects," Front. Heat Mass Transf., 7, 16.

http://dx.doi.org/10.5098/hmt.7.16

Zueco, J., Bég, O.A., Takhar, H.S., and Prasad, V.R., 2009, "Thermophoretic Hydromagnetic Dissipative Heat and Mass Transfer with Lateral Mass Flux, Heat Source, Ohmic Heating and Thermal Conductivity Effects: Network Simulation Numerical Study," Appl. Thermal Eng., 29, 2808-2815. http://doi.org/10.1016/j.applthermaleng.2009.01.015 